(C) 1980. The Genetical Society of Great Britain

\title{
SEQUENCE ORGANISATION IN BARLEY AND OATS CHROMOSOMES REVEALED BY INTERSPECIES DNA/DNA HYBRIDISATION
}

\author{
J. RIMPAU*, D. B. SMITH and R. B. FLAVELL† \\ Department of Cytogenetics, Plant Breeding Institute, Trumpington, Cambridge CB2 $2 L Q$
}

Received 27.vi.79

\begin{abstract}
SUMMARY
The organisation of the repeated and non-repeated sequences in barley and oats genomes has been investigated using the repeated sequences of the wheat, oats, barley and rye genomes as DNA probes. Labelled barley and oats fragments of different lengths (200 to $>7000$ nucleotides were hybridised to the repeated sequence probes and the proportions of the labelled fragments renaturing with the probe DNAs were determined. The average spacings of these sequences through the barley and oats genomes were inferred from the results together with the proportions of the genomes in which the renatured sequences are concentrated. Over 70 per cent of barley and oats DNAs belong to families of repeated sequences. The few copy or non-repeated sequences in these genomes have a mean length of around 700 nucleotide pairs and are interspersed between repeated sequences. These findings have enabled schematic maps of the barley and oats genomes to be drawn. Both genomes appear to be constructed mostly of short sequences with neighbouring sequences being distinguished by their repetition frequency (repeated or nonrepeated) or their homology with different repeated sequence probes. Short non-repeated sequences interspersed with short repeated sequences are concentrated in regions occupying 50 to 60 per cent of the barley genome and 40 to 50 per cent of the oats genome. Most of the remaining DNA consists of tandemly arranged repeated sequences of different evolutionary origins. It is postulated that much of this complex repeated sequence DNA could have arisen from amplification of compound sequences each containing repeated and non-repeated sequences.
\end{abstract}

\section{INTRODUCTION}

IN the last 6 years our understanding of nucleotide sequence organisation in the complex chromosomes of animals has advanced considerably due to the application of new approaches to the problems (Davidson et al., 1973, 1975; and Graham et al., 1974). Experiments describing the general sequence organisation patterns in some higher plant species including cotton (Walbot and Dure, 1976), Nicotiana (Zimmerman and Goldberg, 1978), pea (Murray, Cuellar and Thompson, 1978), soybean (Goldberg, 1978) and parsley (Kiper and Herzfeld, 1978) have also been published. Analyses in this laboratory have concentrated on cereal genomes (Smith and Flavell, 1975, 1977; Flavell and Smith, 1976; Smith, Rimpau and Flavell, 1976; Flavell, Rimpau and Smith, 1977; Rimpau, Smith and Flavell, 1978).

In this paper, the general features of the repeated and non-repeated

* Present address: Institut für Pflanzenbau und Pflanzenzüchtung, Universität der Göttingen, D34, Gottingen, West Germany.

$\dagger$ Author to whom correspondence should be addressed. 
sequences of the oats and barley genomes are described and crude sequence organisation maps of these genomes are derived showing how the nonrepeated and different repeated sequences are interspersed with one another. These maps have been derived from experiments based upon the method first used by Davidson et al. (1973) and Graham et al. (1974) to uncover sequence organisation in the Xenopus and sea urchin genomes. However, to uncover repeated sequence DNA organisation in these cereal species it was necessary to divide the repeated sequences of each genome into groups of unrelated families of repeated sequences. This was achieved by crosshybridisation with the repeated sequence DNAs from other cereal species as described in detail elsewhere (Flavell, Rimpau and Smith, 1977). Analyses of the wheat and rye genomes using these methods have already been published (Rimpau, Smith and Flavell, 1978).

\section{Materials AND Methods}

(i) Isolation of unlabelled and tritium labelled DNAs

Unlabelled DNAs were isolated from young leaves of many plants of Triticum aestivum (wheat), variety Chinese Spring, Secale cereale (rye) variety "Petkus", Hordeum vulgare (barley), variety "Sultan" and from Avena sativa (oats) variety "Maris Titan". The extraction and purification procedures were as described in detail previously (Smith and Flavell, 1974, 1975). The DNA preparations had $\frac{\mathrm{OD}_{260}}{\mathrm{OD}_{230}}>1.9$ and $\frac{\mathrm{OD}_{260}}{\mathrm{OD}_{280}}>1.9$ and more than 98 per cent of the $\mathrm{OD}_{260}$ was retained on hydroxyapatite at $60^{\circ} \mathrm{C}$ in $0 \cdot 12$ м phosphate buffer.

Tritium labelled DNAs from barley and oats were extracted from 3 to 5-day-old seedlings germinated under sterile conditions in the presence of ${ }^{3} \mathrm{H}$ thymidine $\left(\mathrm{CH}_{3}\right.$ labelled $\left.52 \mathrm{Ci} / \mathrm{m} \mathrm{mol}\right)$. The purification method was exactly as described previously (Smith and Flavell, 1974).

\section{(ii) Fractionation of ${ }^{3} \mathrm{H}$ labelled $D \mathcal{N} A$ s and fragment size determinations of labelled and unlabelled DNAs}

The tritium labelled DNAs were sedimented in preparative 5 to 11 per cent $\left(\frac{W}{W}\right)$ linear sucrose gradients in $0.1 \mathrm{~N} \mathrm{NaOH}$ for 19 and 40 hours at 24,000 r.p.m. $(50,000 \times g)$ at $20^{\circ} \mathrm{C}$. The gradients were fractionated and the fractions used as sources of DNA of different fragments sizes. The weight average denatured DNA fragment leng ths in each fraction were subsequently determined by sedimentation in identical gradients together with unlabelled markers as described by Burgi and Hershey (1963). The marker DNAs were sonicated wheat or calf thymus DNAs and their weight average denatured fragment lengths were determined by boundary velocity sedimentation in an MSE Centriscan analytical ultracentrifuge. The order of fractions in the original gradient agreed with the order of sedimentation coefficients subsequently determined for each fraction separately. The values used for average fragment sizes in each fraction were taken from the best-fit line through a plot of fraction number versus sedimentation coefficient.

All unlabelled DNA average denatured fragment lengths were deter- 
mined by sedimentation in $0.9 \mathrm{M} \mathrm{NaCl} 0.1 \mathrm{~N} \mathrm{NaOH}$ in an analycal ultracentrifuge using the equation of Studier (1965).

\section{(iii) Renaturation in vitro and hydroxyapatite chromatography of renaturation products}

For the determination of unlabelled DNA renaturation kinetics, different concentrations of unlabelled DNAs were denatured by incubating at $100^{\circ} \mathrm{C}$ for $5 \mathrm{~min}$. and then incubated at $60^{\circ} \mathrm{C}$ in $0.12 \mathrm{M}$ phosphate buffer $\left(0.18 \mathrm{M} \mathrm{Na}^{+}\right)$ for different time periods. The renatured DNAs were estimated quantitatively by hydroxyapatite chromatography (see below).

In most experiments involving duplex formation between labelled and unlabelled DNA fragments, ${ }^{3} \mathrm{H}$ labelled DNAs of appropriate average fragment size, were added to a large excess (usually ratio $>1: 8000$ ) of unlabelled DNA (see details of individual experiments in Results) sonicated to an average fragment size of 300 to 400 nucleotides. DNAs in $0.12 \mathrm{~m}$ phosphate buffer were denatured at $100^{\circ} \mathrm{C}$ for $5-10 \mathrm{~min}$. before incubation at $60^{\circ} \mathrm{C}$ to the appropriate $\mathrm{C}_{0} \mathrm{t}$ value. $\quad\left(\mathrm{C}_{0} \mathrm{t}=\mathrm{DNA}\right.$ concentration in moles nucleotide per litre $\times$ incubation time in sec.). The samples were then diluted with $0.12 \mathrm{M}$ phosphate buffer and applied to hydroxyapatite columns (Bio Rad HTP) previously equilibrated to $56^{\circ} \mathrm{C}$. DNA eluting at these temperatures was considered non-renatured and DNA in the renatured fraction was recovered by elution with the same buffer at $95^{\circ} \mathrm{C}$.

Unlabelled DNA concentrations in the eluates were determined from $\mathrm{OD}_{260}$ after brief centrifugation to sediment any hydroxyapatite. They were corrected for any $\mathrm{OD}_{260}$ appearing in eluates from columns to which no DNA had been added. Labelled DNA was estimated by precipitating the DNA with trichloracetic acid (final concentration of 5 per cent) in the cold in the presence of $100 \mu \mathrm{g}$ bovine serum albumin and collecting the DNA on glass fibre filters as described elsewhere (Smith and Flavell, 1974; Flavell and Smith, 1976). The recovery and quantitative estimations of labelled DNA by this method is independent of the DNA fragment size over the range used in this work (Flavell and Smith, 1976).

\section{Results}

The renaturation kinetics of oats, barley, wheat and rye DNAs are similar (Smith and Flavell, 1975, 1977; Flavell and Smith, 1976). Essentially all fragments carrying a repeated sequence are included in the renatured DNA fraction isolated by hydroxyapatite chromatography, when denatured DNA is incubated to a $\mathrm{C}_{0} \mathrm{t}$ of 100 at $60^{\circ} \mathrm{C}$ in $0.18 \mathrm{M} \mathrm{Na}+$. Most fragments lacking repeated sequences do not start to renature until a $\mathrm{C}_{0} \mathrm{t}$ exceeding 500 (Smith and Flavell, 1975). Therefore, when these cereal DNAs are denatured and incubated to $\mathrm{C}_{0} \mathrm{t} 100$, the repeated sequences can be easily separated from the non-repeated or few copy sequences by hydroxyapatite chromatography. For convenience, we shall refer to the DNA of cereal DNAs which does not renature by $\mathrm{C}_{0} \mathrm{t} 100$ as "non-repeated DNA" although we recognise it may contain sequences present in a few copies per haploid genome (see Smith and Flavell, 1975).

In a previous paper (Flavell, Rimpau and Smith, 1977) we have shown 
how separate groups of repeated sequences can be distinguished within the barley and oats genomes by hybridising the repeated sequences to the repeated sequences of wheat, rye, barley and oats. The groups of repeated sequences in barley DNA have been named I, II and VI while the groups of repeated sequences of oats have been named I and VII.

The first series of interspecies DNA/DNA hybridisation experiments in this paper were designed to estimate the proportions of each group in the barley and oats genomes and to quantitatively determine the distribution of repeated sequences in each group within these genomes.

\section{(i) Hybridisation of different length barley and oats DNA fragments to repeated sequences of wheat, rye, barley and oats}

Labelled barley/oats DNA of weight average fragment lengths between 200 and 11,000 nucleotides were incubated in turn with greater than 8000 -fold excesses of denatured unlabelled oats, rye, wheat and barley DNA at $60^{\circ} \mathrm{C}$ in $0 \cdot 12 \mathrm{M}$ phosphate buffer $p \mathrm{H} 6.8$ to $\mathrm{C}_{0} \mathrm{t} 120$. The DNAs were fractionated on hydroxyapatite and the proportions of labelled barley/oats DNA in the duplex fraction were determined. The results, corrected for the zero-time binding DNA (see Davidson et al., 1973 and legend to fig. 1) are shown in fig. 1 for barley DNA and fig. 2 for oats DNA.

The proportions of labelled barley and oats DNA in the hydroxyapatite duplex fraction increased with fragment size up to a length of about 3000 nucleotides, especially in the interspecies combinations. For example, in the labelled barley DNA + unlabelled oats DNA curve (fig. 1) at a labelled fragment size of 250 nucleotides, approximately 27 per cent of the barley DNA was in the hydroxyapatite duplex fractions while at a fragment size of 2500 nucleotides, 52 per cent of the barley DNA was in the hydroxyapatite duplex fraction.

From a series of experiments discussed by Davidson et al. (1973), Graham et al. (1974) and Smith, Rimpau and Flavell (1976) it can be concluded that the additional labelled DNA in the duplex fraction with longer fragment lengths is mostly, if not all, single stranded and the proportions of labelled DNA in the renatured configuration are indicated by extrapolations of the curves in figs. 1 and 2 to the ordinate. These proportions are given in tables 1 and 2.

Where only Group I sequences were able to renature (in the labelled barley + unlabelled oats DNA curve of fig. 1 and in the labelled oats + ununlabelled wheat, rye or barley DNA curves of fig. 2), the curves extrapolate to 20 per cent of the barley genome (fig. 1) and 18 per cent of the oats genome (fig. 2). Thus Group I sequences in barley occupy 20 per cent of the genome (table 1) while they occupy approximately 18 per cent of the oats genome (table 2). When Group I and II sequences in the barley genome were able to reanneal (labelled barley + unlabelled wheat or rye DNA curves, fig. 1) the curves extrapolate to approximately 43 per cent of the barley genome. Thus Group II sequences occupy 23 per cent $(43-20)$ of the barley genome. This method of subtraction is legitimate since essentially all Group I sequences in oats are also present in wheat and rye (Flavell, Rimpau and Smith, 1977). When labelled barley DNA was incubated to $\mathrm{C}_{0} \mathrm{t} 120$ with unlabelled barley DNA repeated sequences in Groups I, II and VI renatured and the renatured fraction was 71 per cent of the labelled barley DNA. Thus 


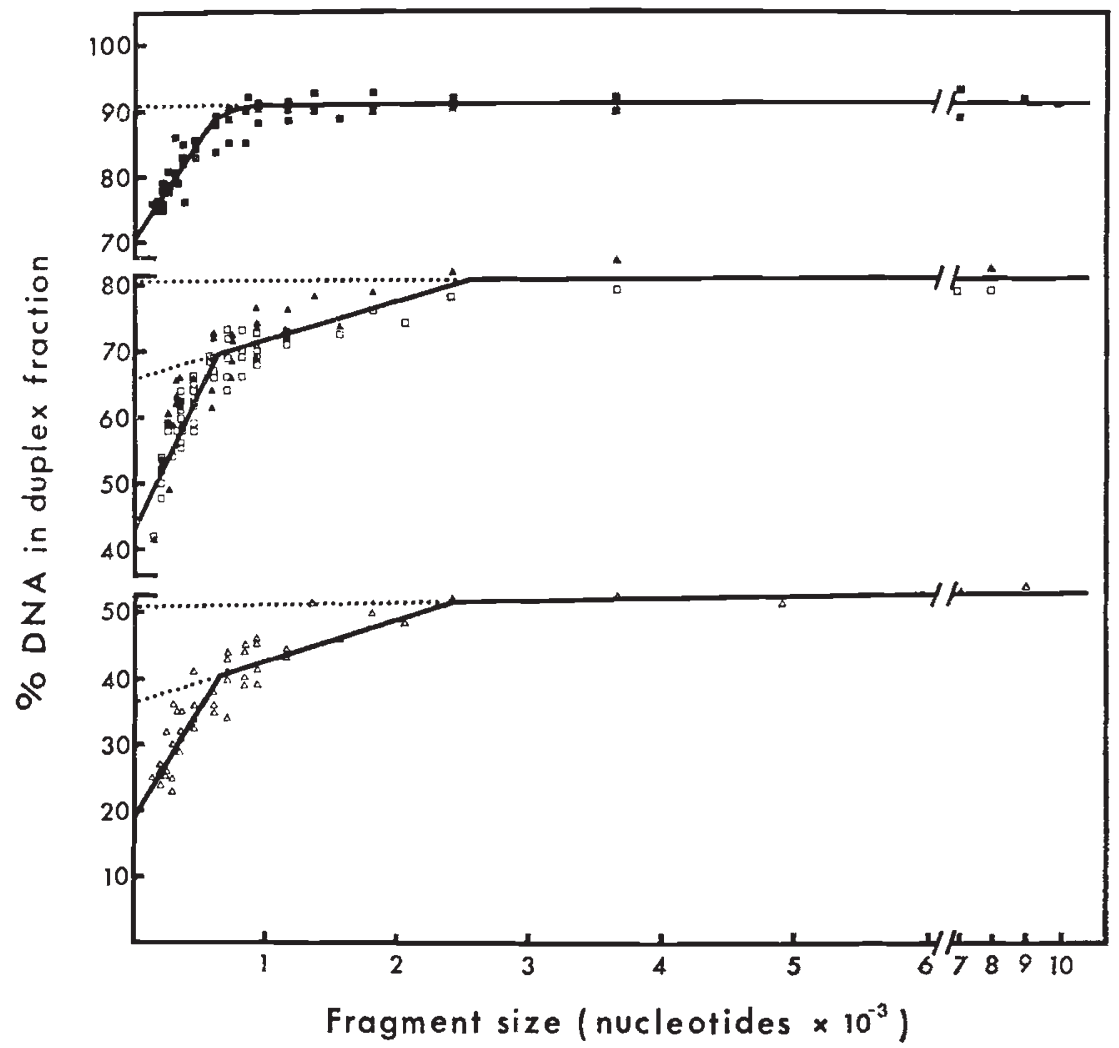

Fig. 1.-Proportion of barley DNA in the hydroxyapatite duplex fraction after hybridisation of different sized fragments to repeated sequences of oats, wheat, rye and barley. ${ }^{3} \mathbf{H}$ labelled barley DNA of various average fragment sizes was mixed with unlabelled oats, wheat, rye or barley DNA of average single stranded fragment size 300 to 400 nucleotides. The concentration of unlabelled DNA was $500 \mathrm{~kg} / \mathrm{ml}$ in $0 \cdot 12 \mathrm{M}$ phosphate buffer and the ratio of labelled to unlabelled DNA exceeded 1:8000. After denaturation and incubation at $60^{\circ} \mathrm{G}$ to $\mathrm{C}_{0} \mathrm{t} 120$, the samples were fractionated on hydroxyapatite. The proportion in the duplex fraction was corrected for the DNA which renatures immediately after denaturing and returning to $60^{\circ} \mathrm{C}$. This correction was as follows:

Corrected \% DNA in duplex fraction =

( $\%$ in HAP duplex fraction $)-(\%$ in zero time HAP duplex fraction) $100-(\%$ in zero time HAP duplex fraction)

The values for $\%$ zero time HAP duplex fractions for each fragment size were taken from results (unpublished) gained exactly as described for the wheat genome (Flavell and Smith, 1976). $\triangle$ hybridisation to oats DNA; $\Delta$ hybridisation to wheat DNA; $\square$ hybridisation to rye DNA; $\square$ hybridisation to barley DNA.

Group VI repeated sequences occupy 28 per cent $(71-43)$ of the barley genome (table 1).

When the repeated sequences in Groups I and VII in the oats genome reannealed (labelled oats + unlabelled oats DNA curve, fig. 2), the curves extrapolated to approximately 73 per cent of the labelled oats DNA. Thus Group VII in oats occupies 55 per cent $(73-18)$ of the oats genome (table 2).

$44 / 1-\mathrm{K}$ 


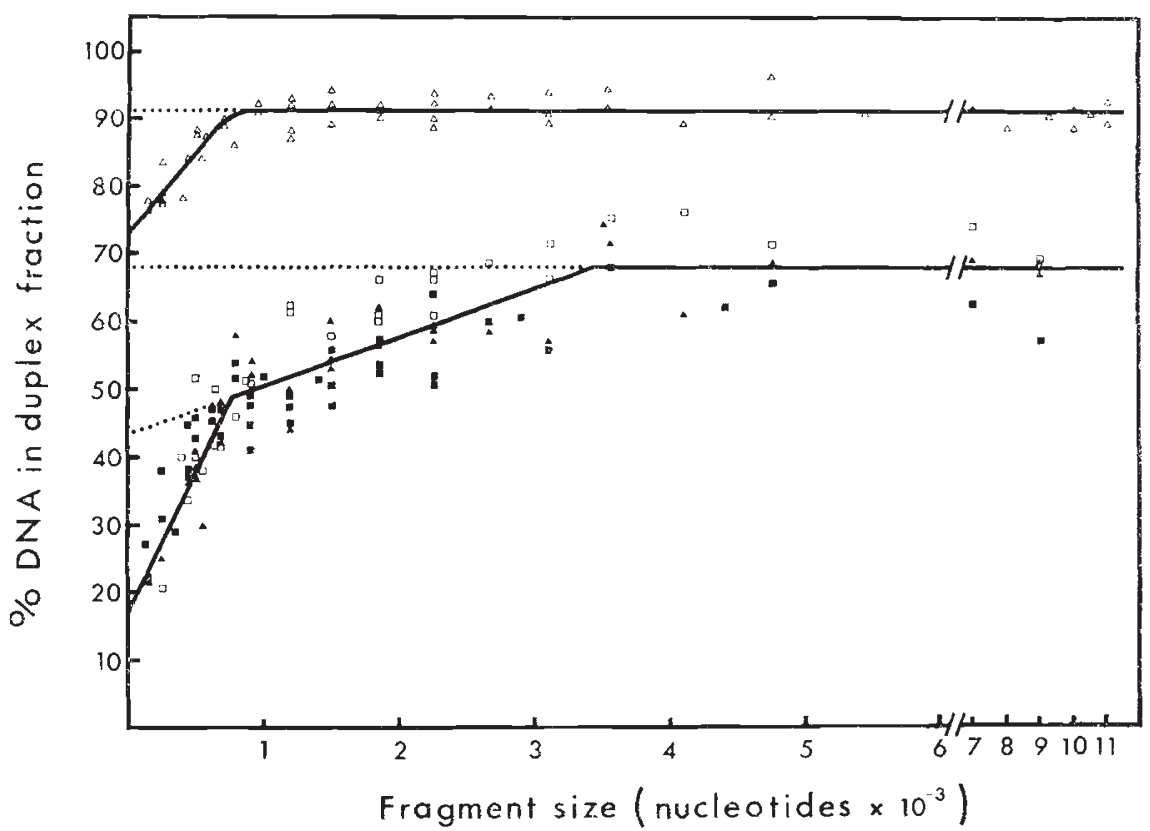

FIG. 2.-Proportion of oats DNA in the hydroxyapatite duplex fraction after hybridisation of different sized fragments to repeated sequences of barley, wheat, rye and oats. The experimental details were as outlined in the legend to fig. 1 for the experiments with labelled barley DNA. A hybridisation to wheat DNA; $\mathbf{n}$ hybridisation to barley DNA; $\square$ hybridisation to rye DNA; $\triangle$ hybridisation to oats DNA.

(ii) Division of the barley and oats genomes into regions containing different repeated sequences

Although the additional labelled DNA in the hydroxyapatite duplex fractions when longer labelled DNA fragments were used in figs. 1 and 2 is single stranded, it is presumably covalently attached to renatured repeated sequences (Flavell, Rimpau and Smith, 1977; Rimpau, Smith and Flavell, 1978). These attached sequences remain single stranded when long fragments (several thousand nucleotides) are used because they are unable to renature under the incubation conditions. The presence of sequences on the same fragment that can and cannot renature under the incubation conditions implies that different sequences are adjacent to one another in the chromosomes. However, because the proportions of the DNAs in the hydroxyapatite duplex fractions (figs. 1 and 2) did not increase when fragments longer than 3500 nucleotides were used, the sequences able to renature under the incubation conditions are generally not greater than 3500 nucleotides apart in the genomes.

When Group I sequences of the barley genome reannealed (labelled barley DNA + unlabelled oats DNA curve, fig. 1) the proportion of the labelled barley DNA in the duplex fraction with fragments longer than 3000 nucleotides was approximately 51 per cent. This implies that the 20 per cent Group I repeated sequences in barley are concentrated in 51 per cent of the barley genome. The chromosomal regions within this 51 per 
TABLE 1

Analysis of homo and heteroduplex curves involving labelled barley DNA (fig. 1)

\begin{tabular}{|c|c|c|c|c|c|}
\hline DNA's incubated together & $\begin{array}{c}\text { Repeated sequence } \\
\text { groups reannealing } \\
\text { and chromosomal } \\
\text { regions in } \\
\text { hydroxyapatite } \\
\text { duplex fraction }\end{array}$ & $\begin{array}{c}\% \text { of genome } \\
\text { renatured } \\
\text { (ordinate intercept) }\end{array}$ & $\begin{array}{l}\% \text { of genome } \\
\text { renatured }+ \text { short } \\
\text { interspersed sequences }\end{array}$ & \multicolumn{2}{|c|}{$\begin{array}{l}\% \text { of genome and } \\
\text { length (in nucleotides) } \\
\text { of non-renatured } \\
\text { spacers between } \\
\text { renatured sequences }\end{array}$} \\
\hline${ }^{3} \mathrm{H}$ barley and unlabelled oats & $\mathrm{I} ; \mathrm{A}$ & $I \stackrel{20^{a}}{=} 20$ & 51 & $\begin{array}{l}17 \\
14\end{array}$ & $\begin{array}{r}650 \\
2500\end{array}$ \\
\hline${ }^{2} \mathrm{H}$ barley and unlabelled rye & $\mathrm{I}+\mathrm{II} ; \mathrm{A}+\mathrm{B}$ & $I I \stackrel{44^{a}}{=} 24$ & 83 & $\begin{array}{l}25 \\
14\end{array}$ & $\begin{array}{r}650 \\
2500\end{array}$ \\
\hline${ }^{8} \mathrm{H}$ barley + unlabelled wheat & $\mathrm{I}+\mathrm{II} ; \mathrm{A}+\mathrm{B}$ & $I I \stackrel{42^{\mathrm{R}}}{=} 22$ & 79 & $\begin{array}{l}23 \\
14\end{array}$ & $\begin{array}{r}650 \\
2500\end{array}$ \\
\hline${ }^{8} \mathrm{H}$ barley + unlabelled barley & $\begin{array}{c}\mathrm{I}+\mathrm{II}+\mathrm{VI} \\
\mathrm{A}+\mathrm{B}+\mathrm{G}\end{array}$ & $\mathrm{VI}^{71^{\mathrm{a}}}=28$ & 91 & 20 & 700 \\
\hline
\end{tabular}

These analyses of fig. 1 were carried out as described in the text.

A small proportion $(₹ 5 \%)$ of this renatured DNA may be self-renatured labelled sequences in excess of $0.25 \times 10^{6}$ copies/ haploid genome. Because this percentage is small and because the homology of the sequences with the DNA from the other species is not known, it has not been subtracted from the estimates of renatured DNA.

TABLE 2

Analysis of homo and heteroduplex curves involving labelled oats DNA (fig. 2)

\begin{tabular}{|c|c|c|c|c|}
\hline DNA's incubated together & $\begin{array}{c}\text { Repeated sequence } \\
\text { groups reannealing } \\
\text { and chromosomal } \\
\text { regions in } \\
\text { hydroxyapatite } \\
\text { duplex fraction }\end{array}$ & $\begin{array}{c}\% \text { of genome } \\
\text { renatured } \\
\text { (ordinate intercept) }\end{array}$ & $\begin{array}{c}\% \text { of genome } \\
\text { renatured }+ \text { short } \\
\text { interspersed sequences }\end{array}$ & $\begin{array}{l}\% \text { of genome and } \\
\text { length (nucleotides) } \\
\text { of non-repeated } \\
\text { spacers between } \\
\text { renatured sequences }\end{array}$ \\
\hline $\begin{array}{l}{ }^{8} \mathrm{H} \text { oats }+ \text { unlabelled barley, } \\
\text { wheat or rye }\end{array}$ & I ; A & $\left(I \stackrel{18^{8}}{=} 18\right)$ & 68 & $\begin{array}{r}750 \\
3400\end{array}$ \\
\hline${ }^{8} \mathrm{H}$ oats + unlabelled oats & $\mathbf{I}+\mathrm{VII} ; \mathrm{A}+\mathrm{H}$ & $(\mathrm{VII}=55)$ & 91 & 750 \\
\hline
\end{tabular}

Results are from mean curves drawn through data points for unlabelled barley, wheat and rye DNAs in fig. 2 .
A small proportion $\left(₹ 5 \%\right.$ ) of this renatured DNA may be self-renatured labelled sequences in excess of $0.25 \times 10^{8}$ copies/ haploid genome. Because this percentage is small and because the homology of the sequences with the DNA from the other species is not known, it has not been subtracted from the estimates of renatured DNA.

cent that contain Group I repeated sequences we have called A regions (Rimpau, Smith and Flavell, 1978). When Group I and II sequences of barley DNA renatured (labelled barley DNA + unlabelled rye or wheat DNA curves, fig. 1), the maximum proportions of labelled barley DNA in the hydroxyapatite duplex fractions were approximately 81 per cent. Thus there are some regions in barley chromosomes amounting to approximately 30 per cent $(81-51)$ of the genome where Group II repeated sequences are present but Group I repeated sequences are not. These we have termed B regions (Rimpau, Smith and Flavell, 1978). When Group I, II and VI repeated sequences renatured (labelled barley DNA + unlabelled barley DNA in the hydroxyapatite duplex fraction was approximately 91 per cent. Thus there are chromosomal regions (termed $\mathrm{G}$ regions) forming 10 per cent $(91-81)$ of the barley genome which possess Group VI repeated sequences but not Group I or II repeated sequences. The 9 per cent of the barley genome that failed to be incorporated into the hydroxyapatite duplex fraction after renaturation of essentially all the barley repeated sequences on very long fragments, presumably consists either of very long arrays of non-repeated sequence DNA (in E regions) or " damaged" DNA unable to reanneal for some reason. The quantitative fractionation of the barley genome into $\mathrm{A}, \mathrm{B}, \mathrm{G}$ and $\mathrm{E}$ regions is depicted in fig. 3. 
Fig. 3.-Fractionation of the barley and oats genome into different regions based upon repeated sequence distribution.

\section{A. The Barley Genome}

Proportion of genome in HAP duplex fraction after renaturation of different groups of repeated sequences.
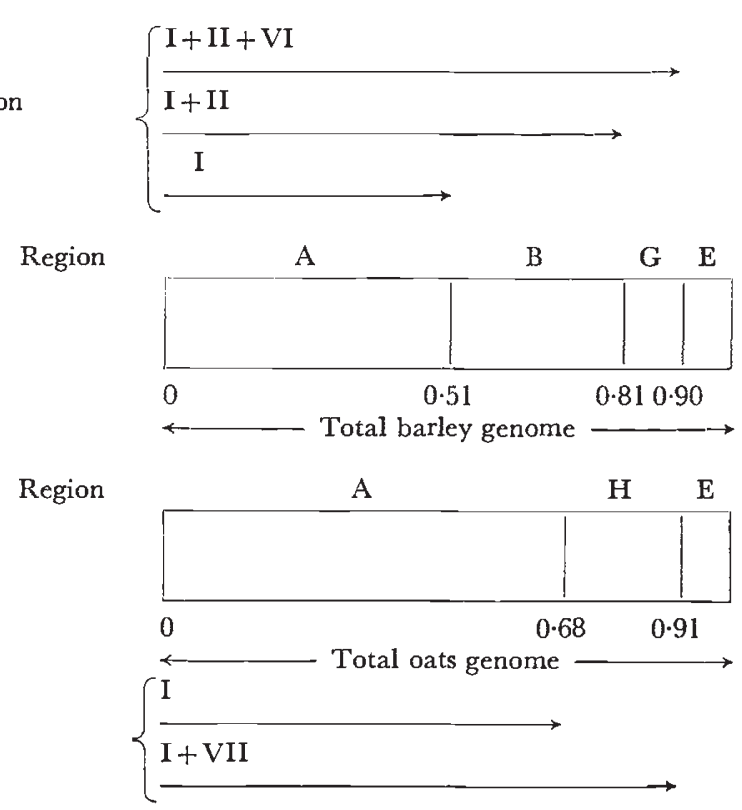

B. The Oats Genome

Proportion of genome in HAP duplex fraction after renaturation of different groups of repeated sequences

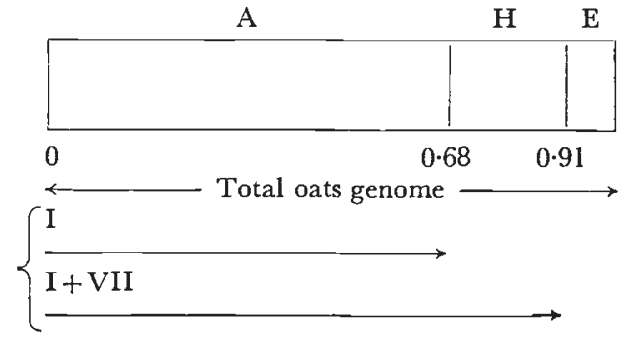

Fractionation of the genomes is based upon the results in figs. 1 and 2, extracted in tables 1 and 2. A regions are defined by the presence of Group I repeated sequences, B regions by the presence of Group II but not Group I repeated sequences, G regions by the presence of the barley-specific Group VI but not Group I or Group II sequences and $\mathrm{H}$ regions by the presence of oats-specific Group VII but not Group I repeated sequences. E regions consist of non-repeated sequences and lack repeated sequences.

When Group I repeated sequences of the oats genome renatured in the labelled oats DNA + unlabelled barley, wheat or rye DNA curves (fig. 2) the maximum proportion of the oats DNA in the hydroxyapatite duplex fraction was 68 per cent. Thus the A regions in which Group I repeated sequences are interspersed in the oats genome form 68 per cent of the genome. When Group I and Group VII repeated sequences reannealed in the labelled oats + unlabelled oats DNA curve (fig. 2), the maximum proportion of the oats DNA in the hydroxyapatite duplex fraction was 91 per cent. Thus 23 per cent $(91-68)$ of the oats genome consists of regions which contain no Group I repeated sequences but do contain Group VII repeated sequences. These regions we have termed $H$ regions. Oats also appears to possess 9 per cent $\mathrm{E}$ regions which lack repeated sequences. The quantitative fractionation of the oats genome into $\mathrm{A}, \mathrm{H}$ and $\mathrm{E}$ regions is depicted in fig. 3.

In the different chromosomal regions defined above, there could be nonrepeated sequences and repeated sequences of groups which are unable to reanneal with the unlabelled DNA. The presence of non-renaturing repeated sequences is deduced later in this paper. The proportions of nonrepeated sequence DNA residing in each of the regions were estimated experimentally. 
(iii) Distribution of non-repeated sequences within each of the chromosomal regions

The proportion of non-repeated sequence DNA in each of the chromosomal regions containing repeated sequences was determined by a method involving two rounds of denaturation and renaturation. The methods and results for barley DNA are illustrated in fig. 4 and for oats DNA in fig. 5.

FIG. 4.-Estimation of non-repeated sequence DNA in the different regions of the barley genome

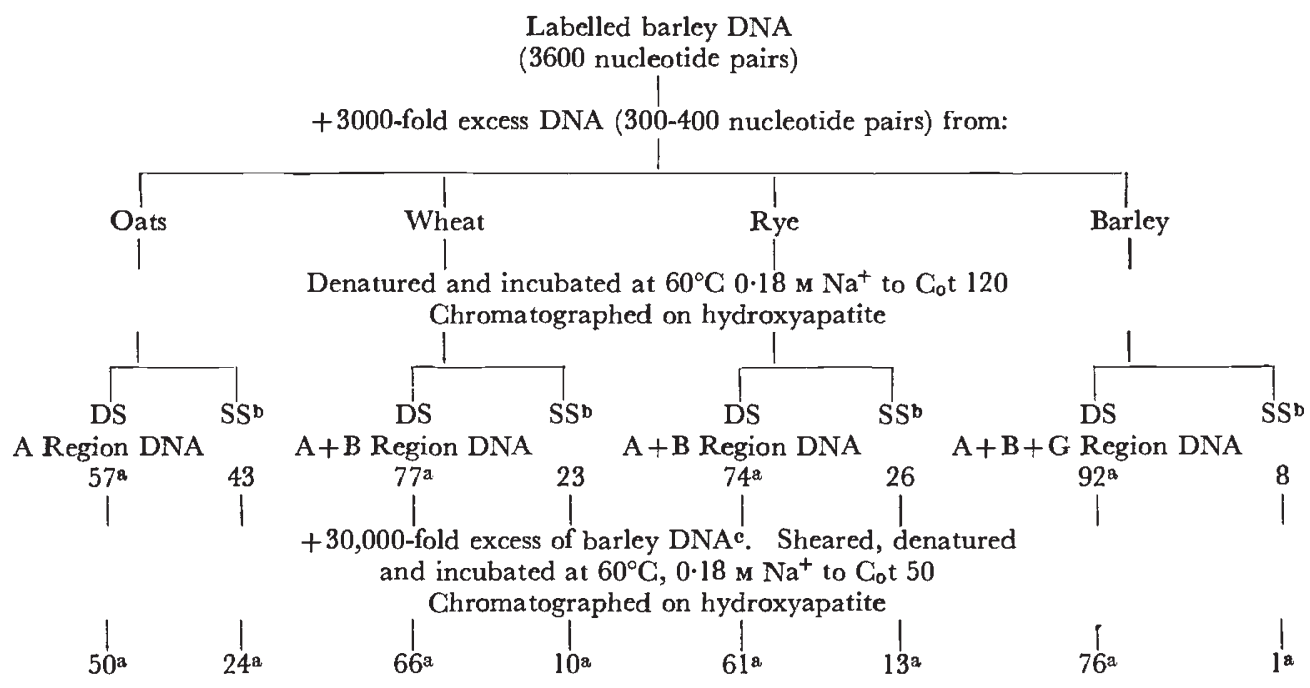

a Percentage of total labelled barley DNA in hydroxyapatite duplex fraction (DS). All percentages are means from two experiments and are corrected for the zero-time binding DNA where appropriate (see legend to fig. 1).

b SS = hydroxyapatite single stranded DNA fraction.

c 30,000-fold excess over the labelled DNA.

In the first part of the procedure, sequences from different chromosomal regions were isolated by hybridising long labelled barley or oats fragments to 3000-fold excesses of short unlabelled DNA fragments of the appropriate species. The isolated long fragments were then sheared to much smaller pieces and the proportions of labelled DNA unable to reanneal to homologous repeated sequences in a second incubation were estimated. These sequences unable to reanneal to homologous repeats were considered as non-repeats, covalently attached to the repeated sequences isolated in the first hybridisation.

When A region DNA was isolated from barley DNA, 7 per cent of the DNA failed to renature to barley repeated sequences after shearing. When $\mathrm{A}+\mathrm{B}$ region barley DNA was isolated, 12 per cent of the DNA (mean of experiments using wheat and rye DNA's to isolate A $+\mathrm{B}$ region DNA) failed to renature with barley repeated sequences after shearing. Sixteen per cent $A+B+G$ region DNA failed to renature to barley repeated sequences after shearing. Thus we conclude that approximately 7 per cent non-repeated sequence DNA is in A regions of the barley genome, 5 per cent (12-7) non-repeated sequence DNA is in B regions and 4 per cent $(16-12)$ is in 
Fig. 5.-Estimation of non-repeated sequence DNA in A and $\mathrm{H}$ regions of the oats genome

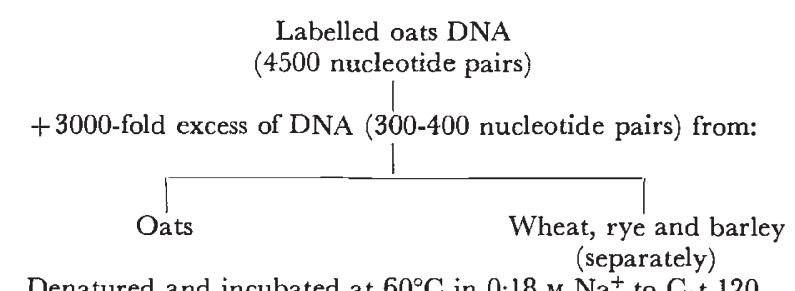

Denatured and incubated at $60^{\circ} \mathrm{C}$ in $0.18 \mathrm{M} \mathrm{Na}^{+}$to $\mathrm{C}_{0} \mathrm{t} 120$
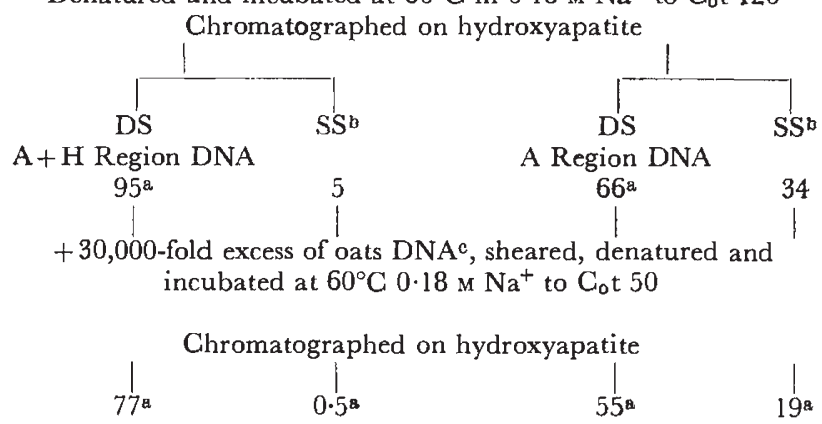

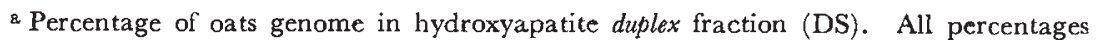
are means from two experiments and are corrected for the zero-time binding DNA where appropriate (see legend to fig. 1).

b SS = hydroxyapatite single stranded DNA fraction.

c 30,000-fold excess over labelled DNA.
}

G regions. There was no evidence for significant damage of labelled DNA during shearing and the two incubations since 76 per cent of the barley DNA was in the hydroxyapatite duplex fraction after the second incubation with unlabelled barley DNA, a value expected for fragments about 250 nucleotides long from the results in fig. 1. Even with fragments of mean length around 250 nucleotides some non-repeated sequence DNA would remain on fragments also containing repeated sequence DNA. From the curve of labelled barley + unlabelled barley DNA in fig. 1, about 5 per cent $(76-71)$ non-repeated sequence DNA would remain on fragments also carrying a repeated sequence. Distributing this 5 per cent within the chromosomal regions, we conclude that 9 per cent $(7+2)$ non-repeated sequence DNA is in A regions, 7 per cent $(5+2)$ is in $B$ regions and 5 per cent $(4+1)$ in $\mathrm{G}$ regions.

In the experiments on oats DNA (fig. 5), 11 per cent of the genome failed to renature when $\mathrm{A}$ region DNA was sheared and reincubated with oats repeated sequence DNA. When $A$ and $H$ region DNA was sheared and reincubated with oats repeated sequence DNA 18 per cent of the oats genome failed to renature. Thus $H$ regions contain 7 per cent $(18-11)$ non-repeated sequence DNA. Again, as indicated for the barley experiments about 4 per cent non-repeated sequence DNA would remain on fragments also containing repeated sequences so we conclude that 13 per cent $(11+2)$ non-repeated sequence DNA is in A regions of the oats genome and 9 per cent $(7+2)$ is in $\mathrm{H}$ regions. 
(iv) Further analysis of figs. 1 and 2 and derivation of sequence organisation units for the regions in barley and oats chromosomes

We have already derived from figs. 1 and 2, the proportions of labelled barley and oats DNAs that renature after hybridisation with the repeated sequences of the different unlabelled DNAs and the proportions of the barley and oats genomes in which these sequences are distributed (see tables 1 and 2). Mean estimates of the lengths of the non-renaturing sequences, which separate the renatured sequences on long fragments, can be estimated from the marked changes of slope in the curves in figs. 1 and 2 (Davidson et al., 1973; Rimpau, Smith and Flavell, 1978). Furthermore, the mean proportions of these spacer sequences can be estimated from extrapolations to the ordinate. For example, from the labelled barley + unlabelled oats DNA curve of fig. 1, it can be concluded that non-renaturing spacer sequences with a mean length of about 650 nucleotides separate the renaturing Group I sequences and these sequences occupy approximately 17 per cent of the barley genome. Also, spacer sequences with a mean length of 2500 nucleotides also separate Group I sequences and occupy 14 per cent of the genome. The results of analysing the curves of figs. 1 and 2 in this way are given in table 1 for the barley genome and table 2 for the oats genome.

Using the parameters defined in tables 1 and 2, and the knowledge of how the groups of repeated sequences and non-repeated sequences are distributed in the different regions defined in fig. 3 , it is possible to infer sequence composition and sequence organisation within the chromosomal regions. It is most convenient to carry out the derivations starting with $\mathrm{E}$ regions and ending with $\mathrm{A}$ regions.

\section{(v) The barley genome}

(a) E regions. These regions consist of long ( $>10,000$ base pairs) nonrepeated sequences and occupy 9 per cent of the genome (organisation unit f, fig. 6).

(b) $G$ regions. These regions occupy 10 per cent of the barley genome (fig. 3) and contain 5 per cent non-repeated sequence DNA (see above). They contain no Group I or Group II repeated sequences so the remaining 5 per cent must consist of Group VI repeated sequences. Since most of the non-repeated sequences appear to have a mean length around 700 base pairs, short non-repeated sequences must be interspersed with short Group VI repeated sequences (organisation unit e, fig. 6) in $\mathrm{G}$ regions.

(c) $B$ regions. These occupy 30 per cent of the genome and also contain 7 per cent non-repeated sequence DNA (see above) which consists of sequences with a mean length of around 700 base pairs. When Group I and II sequences reannealed in the labelled barley + unlabelled rye or wheat DNA curve (fig. 1) the proportion of spacer sequences around 700 nucleotides long increased by approximately 7 per cent compared with when just Group I sequences reannealed in the labelled barley + unlabelled oats DNA curve (fig. 1). This 7 per cent indicates that the short non-repeated sequences reside between Group II sequences in B regions (organisation unit d, fig. 6). The 23 per cent of $B$ region DNA which is not non-repeated must be Group II repeated sequence DNA. 


\begin{tabular}{|c|c|c|c|}
\hline $\begin{array}{l}\text { Chromosome } \\
\text { region†: }\end{array}$ & Unit & Sequence organisation & $\begin{array}{l}\% \text { of each } \\
\text { group }\end{array}$ \\
\hline
\end{tabular}

A

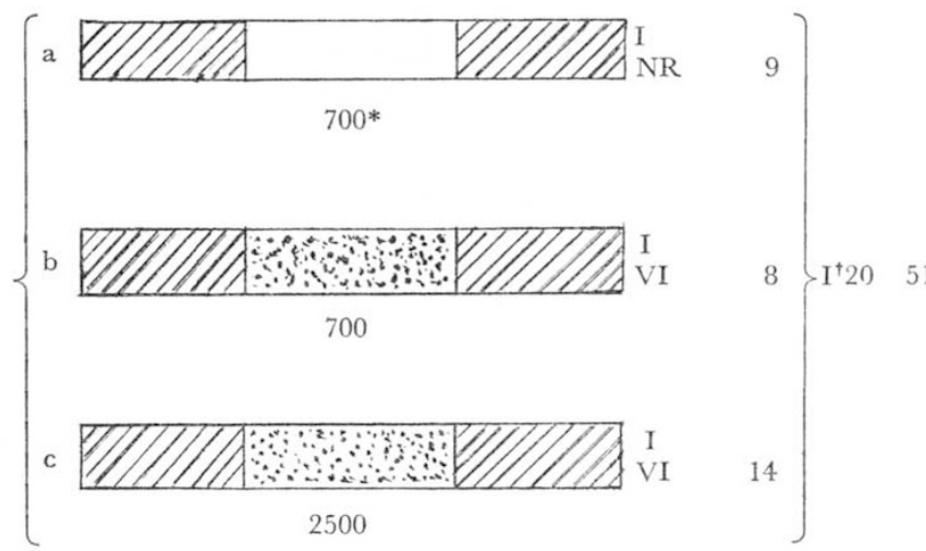

B

d

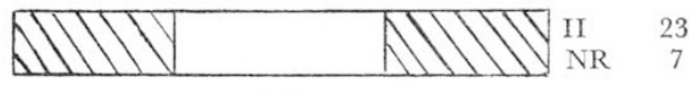

700

G

e

\begin{tabular}{|l|l|l|l|l|l|}
\hline & VI & 5 \\
\hline & &
\end{tabular}

700

E

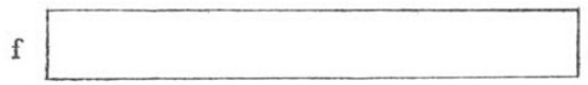
NR $\quad 9$

\section{Group I $\quad$ Group II $\quad \square$ Non-Repeat (NR)}

Fig. 6. -Sequence organisation units in the barley genome

The derivation of the units is described in detail in the text. They account for all the DNA of the genome except the very rapidly renaturing DNA which includes reverse repeats that renature by intrastrand reassociation.

Each of the groups may contain many unrelated families of repeated sequences.

* The numbers under the sequences are the weight average lengths, in nucleotide pairs, as determined from fig. I (see text). The weight average length of group I sequences is probably around 600 nucleotide pairs.

$\dagger$ It is possible that a small proportion of the DNA classified as Group I consists of repeats in excess of $0.25 \times 10^{6}$ copies/haploid genome which self-renatured in the DNA hybridisation experiments used to derive this map (see footnote to table 1). These repeats would probably be clustered in tandem arrays and not closely interspersed with other sequences.

$\ddagger$ These regions are described in fig. 3 . 
(d) A regions. These regions occupy 51 per cent of the genome (fig. 3) and contain approximately 20 per cent Group I repeated sequence DNA and 9 per cent non-repeated sequence DNA in lengths around 700 base pairs interspersed between Group I repeated sequences (organisation unit a, fig. 6). The remaining 8 per cent $(17-9$, table 1$)$ short spacer sequences between Group I sequences in these regions are Group VI repeated sequences, since all Group II DNA is in B regions (organisation unit b, fig. 6). In A regions there are also sequences with mean lengths of 2500 base pairs separating Group I sequences. They occupy 14 per cent of the genome and are not non-repeated. This size spacer class disappeared only in the curve when labelled barley DNA was hybridised with unlabelled barley DNA (fig. 1, table 1). They, therefore, consist of Group VI repeated sequences (sequence organisation unit c, fig. 6).

\section{(vi) The oats genome}

(a) E regions. These consist of long non-repeated sequences ( $>10,000$ base pairs long) occupying 9 per cent of the genome.

(b) $H$ regions. These occupy 23 per cent of the genome (fig. 3) and contain 9 per cent non-repeated sequence DNA in lengths around 750 base pairs long interspersed between Group VII repeated sequences. Since these regions contain no Group I repeated sequences, the only repeated sequences (14 per cent of genome) in these regions are Group VII (organisation unit d, fig. 7).

(c) A regions. These regions occupy 68 per cent of the genome (fig. 3). They contain 18 per cent Group I repeated sequence DNA and 13 per cent non-repeated sequence DNA in lengths around 750 base pairs interspersed between Group I repeated sequences (organisation unit a, fig. 7). The remaining sequences in these regions (37 per cent of genome) must consist of Group VII repeated sequences. Some, occupying 25 per cent of the genome, appear to have a mean length of 3400 base pairs (organisation unit $c$, fig. 7) while the remainder (12 per cent of the genome) appear around 750 base pairs long (organisation unit b, fig. 7). These lengths and percentages come from the curve in fig. 2 , summarised in table 1 .

\section{(vii) Approximate mean lengths of the sequences of the barley and oats genome organisation units}

The methods used to derive the sequence organisation units summarised in figs. 6 and 7 provided estimates of the mean lengths of those sequences which were non-reannealing spacers in the interspersion curves of figs. 1 and 2. These mean lengths are shown in figs. 6 and 7.

The mean length of Group I sequences can be estimated in two ways. First, extrapolation to the abscissa of the labelled barley + unlabelled oats DNA curve in fig. 1 and the labelled oats + unlabelled wheat, rye or barley DNA curve in fig. 2, gives an estimate of the renaturing Group I sequences. This value is about 600 nucleotides for barley and 450 nucleotides for oats. Second, it can be estimated from the number of the spacer sequences separating Group I sequences in the genome. The calculation for the barley genome is as follows. The $1 C$ DNA content of barley is $5.5 \mathrm{pg}$ (Bennett and 
Chromosome Unit

Sequence organisation

$\%$ of each

$\%$ of region +

group

genome

A

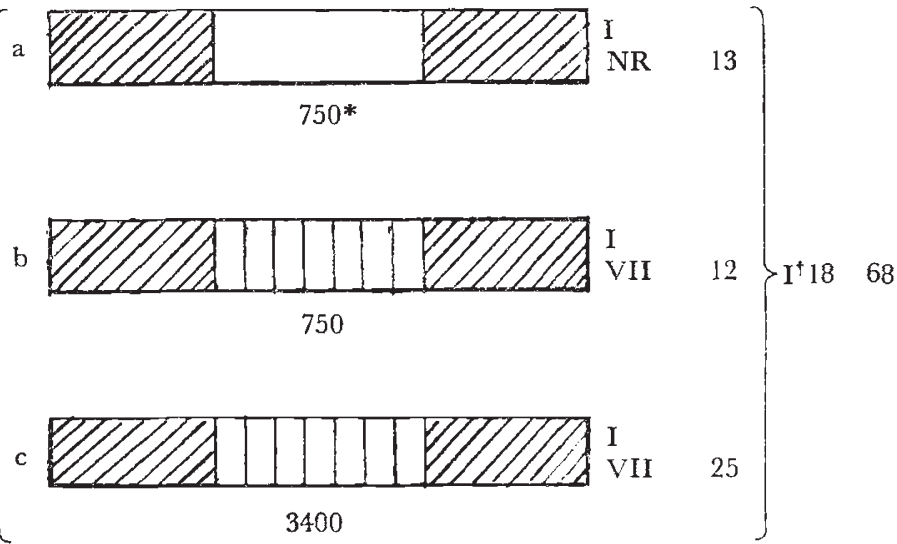

$\mathrm{H}$

$\mathrm{d}$

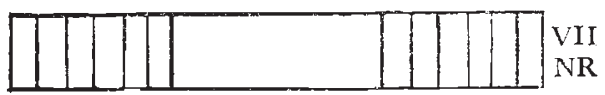

14

9

750

E

e

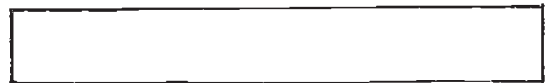

NR

9

9

\section{Group I III] Group VII $\square$ Non-Repeat (NR)}

FIG. 7.-Sequence organisation units in the oats genome

The derivation of the units described in detail in the text. They account for all the DNA of the genome except the very rapidly renaturing DNA which includes reverse repeats that renature by intrastrand reassociation.

Each of the groups may contain many unrelated families of repeated sequences.

* The numbers under the sequences are the weight average lengths, in nucleotide pairs, as determined from fig. 2 (see text). The weight average length of Group I sequences is probably around 450 nucleotide pairs.

$\dagger$ It is possible that a small proportion of the DNA classified as Group I consists of repeats in excess of $0.25 \times 10^{6}$ copies/haploid genome which self-renatured in the DNA hybridisation experiments used to derive this map (see footnote to table 2). These repeats would probably be clustered in tandem arrays and not closely interspersed with other sequences.

$¥$ These regions are described in fig. 3 .

Smith, 1976) which is approximately $5.3 \times 10^{9}$ nucleotide pairs. After subtraction of the 7 per cent very rapidly renaturing DNA not included in the analyses of fig. 1 the DNA content is $4.9 \times 10^{9}$ nucleotide pairs. In fig. 6 , 17 per cent of the DNA consists of sequences with a mean length of 650 to 
700 nucleotide pairs between Group I sequences. There are therefore $\frac{4.9 \times 10^{9} \times 0.17}{650}=1.28 \times 10^{6}$ copies of these per $1 C$ barley genome. In fig. 6,14 per cent of the genome consists of sequences with a mean length of 2500 nucleotide pairs separating Group I sequences. There are therefore $4.9 \times 10^{9} \times 0.14$

$2500-2.7 \times 10^{5}$ copies of these sequences. Assuming there is

one Group I sequence for each spacer sequence, then there are $1.28 \times 10^{6}+$ $2.7 \times 10^{5}=1.6 \times 10^{6}$ Group I sequences per $1 C$ barley genome. Group I DNA accounts for 20 per cent of the genome $=4.9 \times 10^{9} \times 0.20 \times 10^{9}$ nucleotide pairs. The mean length of each Group I sequence is approximately $0.98 \times 10^{9} \div 1.6 \times 10^{6}=600$ nucleotide pairs. This value is in agreement with the value from the extrapolation to the abscissa in fig. 1. The similarly calculated figure for the oats genome assuming a DNA content for oats of $12.7 \times 10^{9}$ nucleotide pairs is 450 nucleotides.

In our studies on the wheat and rye genomes (Rimpau, Smith and Flavell, 1978) we determined experimentally the sizes of Group I sequences in the wheat genome. The weight average size was in the region of 500 base pairs while the number average was around 200 base pairs. This result is in good agreement with the weight average length of Group I sequences of barley and oats derived from the curves in figs. 1 and 2 .

The mean lengths of the Group II sequences in organisation unit $\mathrm{d}$ and Group VI sequences in organisation unit e (fig. 6) of the barley genome can be determined from the proportions of the different sequences in the units and the mean lengths of the non-repeated sequences. The mean length of Group II sequences is given by $23 / 7 \times 700=2300$ nucleotide pairs. The mean length of Group VI sequences is given by $5 / 5 \times 700=700$ nucleotide pairs. These calculations assume an equal number of interspersed nonrepeats and Group II or Group VI repeats in these units. The variation around these means could be considerable.

The average lengths of the Group VII sequences in organisation unit $d$ of the oats genome, calculated similarly is 1150 nucleotide pairs but the variation around this mean could be considerable.

\section{Discussion}

In this paper we have used the repeated sequences of wheat, rye, barley and oats as probes to recognise different groups of repeated sequences in the barley and oats genomes and to distinguish different chromosomal regions, at least 10,000 base pairs long, which do not possess repeated sequences in common (fig. 3). After gaining estimates of the non-repeated sequence DNA within these regions (figs. 4 and 5) it has been possible to produce crude maps of sequence organisation for almost all the DNA in the barley and oats genomes (figs. 6 and 7). However, before discussing the conclusions that can be drawn from these maps, it is important to emphasise the simplifications inherent in the maps and their construction.

First, the lines drawn in figs. 1 and 2 are considered "best fit" straight lines to the experimental points but the point scatter clearly allows for slightly different lines to be drawn. Second, the proportions of renatured DNAs and spacers were gained from extrapolation to the ordinate. Ordinate 
values can vary significantly with relatively small changes in slope. Third, we recognised different spacer units where a marked change in slope occurred. Depending on the relative proportions of different length spacers, where more than one class occurs, it is quite possible for one class of spacers to be masked by others. We chose to distinguish a class of spacer sequences only when a marked change in slope was obvious. Fourth, we have assumed that changes in slope occurred over extremely small changes in fragment length rather than over a range of fragment lengths which is almost certainly more realistic.

These simplifications in interpreting the experiments imply that our derived sizes and proportions of spacer sequences can at best be considered only as mean estimates. We do not have any estimates of the variation around these means. Although the genome maps are therefore schematic and provide only a generalised picture of genome organisation, they do highlight a number of interesting features of these genomes which we now wish to discuss.

\section{(i) The interspersion of short non-repeated sequences and repeated sequences}

The non-repeated sequences of both genomes can be divided into two main classes from the homologous DNA hybridisation curves in figs. 1 and 2 summarised in tables 1 and 2 . One class ( 9 per cent) fails to be incorporated into the hydroxyapatite duplex fraction after renaturation to $\mathrm{C}_{0} \mathrm{t} 120$ because they presumably do not lie close to repeated sequences, i.e., they must be very long ( $>10,000$ nucleotide pairs). It is possible that a small proportion of the 9 per cent fails to renature because it consists of damaged DNA. The other class is incorporated into the duplex fraction when fragments longer than 800 nucleotides were used in the intraspecies hybridisation experiments. This implies that the non-repeated sequences in this class have a mean length of around 700 to 800 nucleotide pairs and are interspersed between repeated sequences. These sequences occupy about 18 to 22 per cent of each of the genomes (see tables 1 and 2 and figs. 6 and 7). Some of the sequences classified as non-repeated at $60^{\circ} \mathrm{C}$ in $0.18 \mathrm{M} \mathrm{Na}^{+}$(figs. 1 and 2) may be highly diverged members of families of repeated sequences.

Non-repeated sequences 600 to 1000 base pairs long are around the length expected for sequences coding for proteins. However, in the haploid barley and oats genomes there appear to be approximately $1 \times 10^{6}$ short nonrepeated sequences, a value considerably in excess of the number of genes likely to be required to code for all the cell functions of a higher plant. It is likely that less than 5 per cent of the short non-repeated sequences are essential gene sequences.

The short non-repeated sequences interspersed with short repeated sequences were found to be concentrated in approximately 57 per cent of the barley genome (fig. 6) and 44 per cent of the oats genome (fig. 7). This interspersion pattern of sequence organisation is common in large proportions of the genome of a very wide range of species (Davidson et al., 1975, 1977; Crain et al., 1976a, b). However, its significance is not understood. It has been suggested that the transcription of non-repeated sequences which specify messenger RNA's may be regulated by neighbouring repeated sequences and more recently Davidson et al. (1977) have speculated that substantial proportions of the genome with this sequence arrangement may 
be transcribed into HnRNA, the HnRNA being cleaved into small fragments which could subsequently control the transcription of unlinked genes. This hypothesis was put forward to provide a role for the relatively large amounts of DNA with this sequence arrangement in which the non-repeats do not specify messenger RNAs. Non-repeated sequence DNA can be formed by extensive sequence alteration of pieces of repeated sequence DNA (Flavell et al., 1980). This may be the origin of some of the short regions of nonrepeated DNA interspersed within repeated DNA Families. However, the extensive interspersion of short pieces of repeated and non-repeated DNA in these cereal genomes also strongly suggests that short pieces of DNA are frequently rearranged in the chromosomes (Flavell et al., 1980). Thus a large fraction of the interspersed short non-repeats and repeats may have no transcribed function but the sequence arrangement reflects the way this kind of DNA is free to evolve in plant chromosomes. A third possibility is that the interspersion pattern may play an important role in chromatin condensation (Lerman and Degtyarev, 1978).

\section{(ii) Interspersion of different short repeated sequences with one another}

An interesting feature emerging from these studies is that the substantial proportions of the genomes consist of different repeats a few hundred base pairs long on average interspersed with one another (units $b$ and $c$ in the barley and oats genome, figs. 6 and 7). This pattern has been uncovered because the interspersed repeats have different evolutionary origins. Similar interspersed repeat patterns occur in the wheat and rye genomes (Rimpau, Smith and Flavell, 1978; Bedbrook et al., 1980; Flavell et al., 1980). The origin of this pattern is particularly interesting. The most likely origin is the amplification of pieces of DNA consisting of two or more sequences of different evolutionary origin and copy number. For example the similarity between the $b$ or $c$ repeated sequence units (figs. 6 and 7) of both genomes and the a units provokes the speculation that the $b$ and $c$ units have arisen from amplification of a units. The a units which consist of non-repeats interspersed with short Group I repeats are likely to have existed in all ancestral forms of oats, barley, wheat and rye since they have repeats common to these four species (Flavell, Rimpau and Smith, 1977). If this speculation is correct then it follows that (1) much of the repeated sequence DNA of these species that has been established in these genomes since the species diverged has been amplified from pieces of DNA over 800 base pairs long, (2) the amplified sequences have part of their unit repeat in common with a pre-existing family of repeated sequences while the other part of their unit repeat is related to non-repeated sequences. Examples of specific repeated sequence DNA families with these properties have recently been studied in detail (Bedbrook, O'Dell and Flavell, unpublished).

The complexity of the arrangement of the interspersed repeated sequences would explain why so little of these DNAs separated into simple sequence "satellites" in buoyant density gradients (Huguet and Jouanin, 1972; Ranjekar et al., 1976). The generality of this kind of repeated sequence organisation in other large plant genomes remains to be more fully elucidated. Small fractions of the mouse and human genomes have been reported to possess apparently similar repeated sequence arrangements (Gech and Hearst, 1976; Marx et al., 1976). 
An important question, in considering the linear organisation of complex repeated sequences, is " are members of the same family clustered in the genome or finely interspersed with sequences of other families?". Recent studies have provided direct evidence for the clustering of identical complex repeated sequences in rye (Bedbrook et al., 1980). Clustering of identical repeats is expected because it is difficult to envisage how sequence amplification occurs without producing tandem arrays of the repeats. However, translocation of blocks of sequences is probably also a frequent event in evolution. The distribution of specific families of repeated sequences in these genomes is currently being studied in our laboratory by in situ hybridisation of purified individual repeated sequences (Bedbrook et al., 1980).

\section{Congluding Remarks}

The utilisation of repeated sequences of related species as probes to uncover sequence organisation has enabled us to conclude that most of each of these complex plant genomes consists of relatively short, distinct sequences. This structure has been recognised because the short sequences interspersed with one another differed either in frequency (non-repeat or repeat) or in evolutionary origin (Groups I, II, VI or VII). The general, schematic genome maps do not illustrate the detailed arrangement of specific repeats such as those in rDNA, which occupy only small proportions of the genomes. Nor do they incorporate the reverse repeats (Flavell and Smith, 1976; Smith and Flavell, 1977) or describe the arrangements of simple sequence satellite DNA's. Details on the sequence arrangements of specific repeats is clearly best studied for each particular repeat. However, for large complex genomes it is clearly essential that more broad details of sequence organisation, such as those reported here, are accumulated so that some of the general questions about chromosome organisation and behaviour can be discussed and answered.

This paper analysing the barley and oats genomes complements a similar analysis of the wheat and rye genomes (Rimpau, Smith and Flavell, 1978). Comparisons of the four genomes with respect to their repeated sequences (Flavell, Rimpau and Smith, 1977) and the arrangements of these sequences in the genomes gives some insight into the changes that have taken place in the DNA of the species since their divegence from a common ancestor. These changes have recently been summarised (Flavell et al., 1980).

\section{REFERENGES}

BEDBROOK, J. R., JONES, J., O'DELL, M., THOMPSON, R. D., AND FLAVELL, R. B. 1980. A molecular description of telomeric heterochromatin in Secale species. Cell (in press)

Bennetr, m. D., AND smith, J. B. 1976. Nuclear DNA amounts in angiosperms. Phil. Trans. Royal Society (London) B., 274, 227-274.

BURGI, E., AND HERSHEY, A. D. 1963. Sedimentation rate as a measure of molecular weight of DNA. Biophysical 7., 3, 309-321.

CECH, T. R., AND HEARST, J. E. 1976. Organisation of highly repeated sequences in mouse main band DNA. 7. Mol Biol., 100, 227-256.

CRAIN, W. R., DAVIDSON, E. H., AND BRITTEN, R. J. 1976a. Contrasting patterns of DNA sequence arrangement in Apis mellifera (honeybee) and Musca domestica (housefly). Chromosoma (Berl.), 59, 1-12.

CRAIN, W. R., EDEN, F. C., PEARSON, w. R., DAVIDSON, E. H., AND BRITTEN, R. J. (1976b). Absence of short period interspersion of repetitive and non-repetitive sequences in Drosophila DNA. Chromosoma (Berl.), 56, 309-326. 
DAVidson, E. H., galau, G. A., ANgerer, R. C., AND britten, R. 1975. Comparative aspects of DNA organisation in Metazoa. Chromosoma (Berl.), 51, 253-259.

DAVIDson, E. H., HOUGH, B. R., AMENSON, C. S., AND BRITTEN, R. J. 1973. General interspersion of repetitive with non-repetitive sequence elements in the DNA of Xenopus. 7. Molec. Biol., 77, 1-23.

DAVIDSON, E. H., KLEIN, W. H., AND BRITTEN, R. J. 1977. Sequence organisation in animal DNA and a speculation on $\mathrm{h}_{\mathrm{n}} \mathrm{RNA}$ as a coordinate regulatory transcript. Dev. Biol., $55,69-84$.

FLAVELL, R. B., RIMPAU, J., AND SMITH, D. B. 1977. Repeated sequence DNA relationships in four cereal genomes. Chromosoma (Berl.), 63, 205-222.

FLAVELL, R. B., RIMPAU, J., SMITH, D. B., o'DELL, M. AND BEDBROoK, J. R. 1980. The evolution of plant genome structure. In Plant Genome Organisation and Expression, ed. C. J. Leaver. Plenum Press (in press).

FLAVELL, R. B., AND SMITH, D. B. 1976. Nucleotide sequence organisation in the wheat genome. Heredity, 37, 231-252.

GOLDBERG, R. B. 1978. DNA sequence organisation in the soybean plant. Biochemical Genetics, 16, 45-68.

GRAHAM, D. E., NEUFELD, B. R., DAVIDSON, E. H., AND BRITTEN, R. J. 1974. Interspersion of repetitive and non-repetitive DNA sequences in the sea urchin genome. Cell, 1, 127-137

Huguet, r., AND JouAnin, L. 1972. Wheat DNA: Study of the heavy satellite in $\mathrm{Ag}+\mathrm{Cs}_{2} \mathrm{SO}_{4}$ density gradients. Biochim Biophys Res. Comm., 46, 1169-1174.

KIPER, M., AND HERZFELD, F. 1978. DNA sequence organisation in the genome of Petroselinum sativum. Chromosoma (Berl.).

LERMAn, M. 1., AND Degtyarev, s. v. 1978. Periodically interspersed repetitive sequences may govern higher-order DNA coiling in chromatin and chromosomes. Molec. Biol. Rep., 4, 117-120.

MARX, K. A., ALLEN, J. R., AND HEARST, J. E. 1976. Characterisation of the repetitious human DNA families. Biochim. Biophys. Acta., 425, 129-147.

MURRAY, M. G., CUELlaR, R. E., AND THOMPSON, W. F. 1978. DNA sequence organisation in the pea genome. Biochemistry, 17, 5781-5790.

RANJEKAR, P. K., PALlotTA, D., AND LEFonTAine, J. G. 1976. Characterisation of repetitive DNA in barley and wheat. Biochim. Biophys. Acta., 425, 30-40.

RIMPAU, J., SMITH, D. B., AND FLAVELL, R. B. 1978. Sequence organisation analysis of the wheat and rye genomes by interspecies DNA/DNA hybridisation. F. Molec. Biol., 123, 327-359.

SMITH, D. B., AND FLAVELL, R. B. 1974. The relatedness and evolution of repeated nucleotide sequences in the genomes of some Gramineae species. Biochem. Genetics, 12, 243-256.

SMTTH, D. B., AND FlAVEll, R. B. 1975. Characterisation of the wheat genome by renaturation kinetics. Chromosoma (Berl.), 50, 223-242.

SMTTH, D. B., AND FLAVELL, R. B. 1977. Nucleotide sequence organisation in the rye genome. Biochim Biophys Acta., 474, 82-97.

SMITH, D. B., RIMPAU, J., AND FLAVELL, R. B. 1976. Interspersion of different repeated sequences in the wheat genome revealed by interspecies DNA/DNA hybridisation. Nucleic Acid Research, 3, 2811-2825.

studier, F. w. 1965. Sedimentation studies of the size and shape of DNA. F. Molec. Biol., $11,373-390$.

WALBot, v., AND DURE, L. s. 1976. Developmental biochemistry of cotton seed embryogenesis and germination. 7. Mol. Biol., 101, 503-536.

ZIMMERMAN, J. L., AND GOLDBERG, R. B. 1977. DNA sequence organisation in the genome of Nicotiana tabacum. Chromosoma (Berl.), 59, 227-252. 